\title{
A Budget Impact Analysis of Substituting Sitagliptin with Liraglutide in Type 2 Diabetes from A Private Health Insurance Perspective in Egypt
}

Gihan Hamdy El-sisi ( $\nabla$ gihanhamdyelsisi@hotmail.com )

Cairo University https://orcid.org/0000-0002-2917-9388

Ayman Afify

Novo Nordisk

Ashraf Abgad

Alexandria University

Ibtissam Zakaria

Cairo University

Nabil Nasif

Ain Shams University

Hani Naiem Ibrahim

diabetes clinic

Nabil Raafat

Al-Azhar University

joao Carapinha

North-Eastern Hill University

\section{Research}

Keywords: metformin, pioglitazone, gliclazide, hospitalizations

Posted Date: August 11th, 2021

DOl: https://doi.org/10.21203/rs.3.rs-772763/v1

License: (c) (1) This work is licensed under a Creative Commons Attribution 4.0 International License.

Read Full License 


\section{Abstract}

\section{Introduction}

Type 2 diabetes mellitus (T2DM) causes a sizable burden globally both from health and economic points of view.This study aimed to assess the budget impact of substituting sitagliptin with liraglutide versus other glucose lowering drugs from the private health insurance perspective in Egypt over a 3-year time horizon.

\section{Methods}

Two budget impact models were comparedthe standard of care (metformin, pioglitazone, gliclazide, insulin glargine, repaglinide, and empagliflozin)administered in addition to liraglutide or sitagliptin versus the standard of care with placebo. A gradual market introduction of liraglutide or sitagliptin was assumed, and the existing market shares for the other glucose lowering drugs were provided and validated by Expert Panel. The event rates were extracted from the LEADER and TECOS trials. Direct and mortality costs were measured. Sensitivity analyses were performed.

\section{Results}

The estimated target population of 120,574 T2DM adult patients were associated with CV risk. The budget impact per patient per month (PPPM) for liraglutide is EGP29 (\$6.7), EGP39 (\$9), and EGP49 (\$11.3) in the first, second, and third year, respectively. The budget impact PPPM for sitagliptin is EGP11 (\$2.5), EGP14 (\$3.2), and EGP18 (\$4.1) in the first, second, and third year, respectively. Furthermore, adoption of liraglutide resulted in 203 fewer deaths and 550 avoided hospitalizations, while sitagliptin resulted in 43 increased deaths and 14 avoided hospitalizations. The treatment costs of liraglutide use are mostly offset by substantial savings due to fewer CV-related events, avoided mortality and avoided hospitalizations over 3-years.

\section{Conclusion}

Adding liraglutide resulted in a modest budget impact, suggesting that the upfront drug costs were offset by budget savings due to fewer CV-related complications and deaths avoided compared to the standard of care. While sitagliptin resulted in a small budget impact but associated with deaths increased and less hospitalizations avoided.

\section{Key Points}

The upfront drug costs of adding liraglutide were offset by its budget savings due to fewer CV-related complications and deaths avoided compared to the standard of care (modest budget impact). While sitagliptin resulted in a small budget impact but associated with deaths increased and less hospitalizations avoided. This study will help to guide the reimbursement decisions in Egypt. 


\section{Introduction}

Diabetes mellitus is a chronic disease characterized by high levels of plasma glucose and deficiency in insulin production or utilization. Diabetes causes a sizable burden globally both from health and economic points of view. In 2017, they estimated that the number of people suffering from diabetes worldwide is 425 million, a number that is expected to increase to 629 million by 2045 [1]. Of the current diabetic population, $79 \%$ are from low- and middle-income countries, and the highest prevalence is among people aged 40 and 59 [1]. Diabetes costs public health systems around the world about 727 billion USD and caused 4 million deaths in 2017 [1].

The International Diabetes Federation (IDF) ranked Egypt as ninth highest country in the number of type 2 diabetes mellitus (T2DM) patients [2]. The number of T2DM patients is increased threefold over 20 years ago with a current prevalence of $15.6 \%$ among age group 20 to 79 [2]. T2DM is a metabolic disease associated with microvascular and macrovascular complications. The control of plasma glucose levels is associated with reduced risk of microvascular complications (retinopathy, nephropathy, and neuropathy), and benefits for macrovascular complications (reduced rates of heart attacks, strokes and improved blood flow to legs) [3].

Liraglutide (approved in Egypt for treating T2DM) is a human glucagon like peptide 1 (GLP-1) with an established plasma glucose lowering effect, thus reducing the risk of microvascular complications [3]. The effects of glycemic control on macrovascular complications were evaluated in the LEADER clinical trial [3]. The double-blind randomized trial, included 9,340 participants at randomization to investigate the cardiovascular safety of liraglutide versus the standard of care (metformin, thiazolidinedione, sulfonylureas, insulin, meglitinides and FGABG (SGLT2i)) in T2DM patients with high risk for cardiovascular (CV) events. The trial concluded that the rate of cardiovascular complications (nonfatal myocardial infarction (MI), nonfatal stroke) and all cause death were lower in the liraglutide group compared to the standard of care [3]. The TECOS trial is a randomized, double-blind study, assigned 14,671 patients to add either sitagliptin or placebo to their existing therapy [4]. The median follow-up was 3 years to assess the cardiovascular implication to adding sitagliptin to the standard of care for T2DM patients. The primary composite outcome for this non-inferiority trial was CV death, nonfatal stroke, nonfatal MI and hospitalization for unstable angina. Sitagliptin was found noninferior to placebo [4].

This study assessed the budget impact of substituting sitagliptin with liraglutide versus other glucose lowering drugs (metformin, pioglitazone, gliclazide, insulin glargine, repaglinide, and empagliflozin) from the private health insurance perspective in Egypt over a 3-year time horizon.

\section{Methodology}

\subsection{Population and treatment mix}

Two budget impact models were constructed. The first one assessed the budget impact of the use of liraglutide versus other glucose lowering drugs while the second model assessed the budget impact of 
the use of sitagliptin versus other glucose lowering drugs. The target population of T2DM patients was estimated with the current Egyptian adult population and the prevalence of T2DM in Egypt $[2,5]$. The estimated target population was then narrowed to a group of diagnosed and treated patients at risk of cardiovascular events as demonstrated in Fig. 1 [6]. This study focused exclusively on the proportion of this patient group covered by private health insurance companies [7]. The number of targeted patients was estimated to be 120,574. A gradual market introduction of liraglutide or sitagliptin was assumed, and the existing market shares for the other glucose lowering drugs were provided and validated by Expert Panel.

\subsection{Clinical Parameters}

The event rates for cardiovascular complications were extracted from the LEADER trial [3]. The events considered in this study included mortality, myocardial infarction, stroke, heart failure [HF], coronary revascularization, and microvascular complications (retinopathy, and nephropathy). The event rate difference per complication (liraglutide vs. standard of care) was multiplied by the number of patients on liraglutide to determine the number of cardiovascular events avoided. Similarly, sitagliptin inputs were extracted from the TECOS trial and all clinical parameters were included in the model (Table 1). 
The Model inputs parameters

$\begin{array}{llll}\text { Parameter } & \begin{array}{l}\text { Base } \\ \text { Value }\end{array} & \text { Low Value High Value Source }\end{array}$

Population data

Total Adult Population

T2DM prevalence

Proportion of patients with Type 2 Diabetes diagnosed \& treated (with CV morbidity)

Proportion of patients covered by private insurance companies in Egypt

GLP-1RA Adoption year 1

GLP-1RA Adoption year 2

GLP-1RA Adoption year 3

Clinical parameters from the LEADER Trial.

\section{$M{ }^{*}$}

Ischemic Stroke*

HF (hospitalization)*

Coronary Revascularization*

Retinopathy*

Nephropathy*

Unstable Angina Pectoris (hospitalization)*

All-cause mortality*

Clinical parameters from the TECOS Trial.

$\mathrm{MI}^{\#}$

Ischemic Stroke\#
$50,364,992 \quad 40,291,994 \quad 60,437,990 \quad[5]$

$13.7 \%$

$10.9 \%$

$28 \%$

$16.4 \%$

[2]

$35 \%$

$42 \%$

[6]

$5 \%$

$4 \%$

$6 \%$

[7]

$3 \%$

$2.4 \%$

$3.6 \%$

IMS

data

$4 \%$

$3.2 \%$

$4.8 \%$

IMS

data

$5 \%$

$4 \%$

$6 \%$

IMS

data

\begin{tabular}{l} 
[5] \\
\hline 2$]$ \\
\hline 6$]$ \\
\hline 7$]$ \\
IMS \\
data \\
IMS \\
data \\
IMS \\
data
\end{tabular}




\begin{tabular}{|c|c|c|c|c|}
\hline Parameter & $\begin{array}{l}\text { Base } \\
\text { Value }\end{array}$ & Low Value & High Value & Source \\
\hline Unstable Angina Pectoris (hospitalization) ${ }^{\#}$ & $-0.20 \%$ & -0.00160 & -0.00240 & [4] \\
\hline Severe hypoglycemia\# & $0.30 \%$ & 0.00240 & 0.00360 & [4] \\
\hline CV Death\# & $0.30 \%$ & 0.00240 & 0.00360 & [4] \\
\hline Acute pancreatitis\# & $0.10 \%$ & 0.00080 & 0.00120 & [4] \\
\hline HF (hospitalization) \# & $0.10 \%$ & 0.00080 & 0.00120 & [4] \\
\hline Pancreatic cancer $\#$ & $-0.10 \%$ & -0.00080 & -0.00120 & [4] \\
\hline \multicolumn{5}{|l|}{ Treatment Costs per unit (Egyptian Pounds) } \\
\hline Liraglutide & 87.60 & 70.08 & 105.12 & [8] \\
\hline Sitagliptin 100 & 11.00 & 8.80 & 13.20 & [8] \\
\hline Metformin 1000 & 0.90 & 0.72 & 1.08 & [8] \\
\hline Insulin (Glargine) $100 \mathrm{IU}$ & 126.00 & 100.80 & 151.20 & [8] \\
\hline SU (Gliclazide 60 mg) & 1.58 & 1.26 & 1.89 & [8] \\
\hline TZD (Pioglitazone 15mg) & 5.13 & 4.11 & 6.16 & [8] \\
\hline Novonorm 2 mg (Repaglinide) & 1.47 & 1.17 & 1.76 & [8] \\
\hline SGLT2i (Empagliflozin) & 16.53 & 13.23 & 19.84 & [8] \\
\hline \multicolumn{5}{|c|}{ Event Costs excluding medicines (in Egyptian Pounds) } \\
\hline Non-fatal MI & 76719 & 61375 & 92062 & [8] \\
\hline Non-fatal Stroke & 65928 & 52742 & 79113 & [8] \\
\hline HF (hospitalization) & 161249 & 128999 & 193498 & [8] \\
\hline Coronary & 67919 & 54335 & 81502 & [8] \\
\hline \multicolumn{5}{|l|}{ Revascularization } \\
\hline Retinopathy & 20000 & 16000 & 24000 & [8] \\
\hline Nephropathy & 215695 & 172556 & 258834 & [8] \\
\hline Unstable Angina Pectoris (hospitalization) & 76719 & 61375 & 92062 & [8] \\
\hline \multicolumn{5}{|c|}{ *rate difference with and without liraglutide, \#rate difference with and without sitagliptin } \\
\hline \multicolumn{5}{|c|}{$\begin{array}{l}\text { T2DM: type } 2 \text { diabetes mellitus, CV: cardiovascular, GLP1-RA; Glucagon like peptide } 1 \text { receptor } \\
\text { agonist, HF: heart failure, MI: myocardial infarction, SU: sulphonyl urea, TZD: thiazolidinediones, } \\
\text { SGLT2i: Sodium/glucose cotransporter-2 inhibitors }\end{array}$} \\
\hline
\end{tabular}




\begin{tabular}{|lllll}
\hline Parameter & $\begin{array}{l}\text { Base } \\
\text { Value }\end{array}$ & Low Value & High Value & Source \\
\hline Acute Pancreatitis & 55883 & 44706 & 67059 & {$[8]$} \\
\hline Severe Hypoglycemia & 8059 & 6447 & 9670 & {$[8]$} \\
\hline Pancreatic cancer & 92136 & 73708 & 110563 & {$[8]$} \\
\hline Mortality & 750,000 & 600,000 & 900,000 & {$[8]$} \\
\hline *rate difference with and without liraglutide, \#rate difference with and without sitagliptin & \\
\hline $\begin{array}{l}\text { T2DM: type 2 diabetes mellitus, CV: cardiovascular, GLP1-RA; Glucagon like peptide 1 receptor } \\
\text { agonist, HF: heart failure, Ml: myocardial infarction, SU: sulphonyl urea, TZD: thiazolidinediones, }\end{array}$ \\
\begin{tabular}{l} 
SGLT2i: Sodium/glucose cotransporter-2 inhibitors \\
\hline
\end{tabular}
\end{tabular}

\subsection{Costs}

Direct medical costs were estimated for drug acquisition, drug administration, complication management, follow up and adverse events costs. All unit costs of medications were extracted from the private health insurance payer's lists, as fixed reimbursement amount, and multiplied by the drug utilization to obtain monthly and annual costs for liraglutide, sitagliptin, metformin, thiazolidinediones, sulfonylureas, insulin, meglitinides and SGLT2i. The drug utilization proportions were extracted from the LEADER and TECOS trials $[3,4]$.

The average management cost for a $\mathrm{Ml}$ and unstable angina included cost of diagnostic tests (CT scan and ECG test), either percutaneous coronary intervention (PCI) or Coronary artery bypass graft (CABG) intervention, and a 7-day hospital stay including appropriate treatment. The management cost for stroke included a brain magnetic resonance imaging (MRI), 6 days in the ICU and 6 days in general ward including appropriate treatment. The heart failure management costs composed of diagnostic tests (echo doppler and ECG tests), 4 days in the ICU and 10 days in general ward costs including appropriate treatment. The costs of retinopathy included corrective surgery and measures for better glycaemic control, and the costs of nephropathy included measures to control blood pressure, haemodialysis to counteract the kidney damage, and glomerular filtration rate (GFR) test every three months.

The drug related adverse events included were acute pancreatitis, pancreatic cancer, and severe hypoglycaemia [3, 4]. Pancreatitis management costs composed of the cost of 7 days in the intensive care unit (ICU), 3 days of hospitalization in a ward, pain killers and antibiotic. As for the average management cost of pancreatic cancer it was assumed to be limited to the annual price of erlotinib, an epidermal growth factor receptor (EGFR) tyrosine kinase inhibitor approved for the management of pancreatic cancer, which is given daily via oral route. The cost of severe hypoglycaemia management was calculated as the summation of 4 days of hospitalization, admission to the emergency room, glucose intravenous administration, and laboratory tests (creatinine test, GFR tests, liver function tests, and glucose tests). All unit costs were extracted from the private insurance hospitals [8]. 
The mortality costs were also considered in our analysis. Even though indirect costs are not typically included in budget impact analysis, according to the ISPOR task force for good research practices for budget impact analysis, we considered the mortality as the private insurance companies have to pay compensation to the patients' family in case of death based on the contracted life insurance policies [9]. To measure the cost of mortality, each patient life was estimated to have the stated value mentioned in the life insurance policy agreements.

The total event cost for each complication was multiplied by the event rate in each treatment arm (from the LEADER and TECOS trials) to determine the total cost per event. The medication costs were included with event costs, and the total cost difference of liraglutide vs without liraglutide was calculated as the budget impact of adopting liraglutide in T2DM patients with cardiovascular risks from the private health insurance perspective in Egypt. Similarly, the budget impact of sitagliptin vs without sitagliptin was calculated in T2DM patients with cardiovascular risks from the private health insurance perspective. Both liraglutide and sitagliptin were compared to placebo/standard of care. No direct comparison was made between the two interventions. All unit costs in this study were calculated in Egyptian Pounds (EGP) set in 2020 and were exchanged to USD using purchasing power parity rate. The time horizon for the study was 3 years.

\subsection{Sensitivity Analyses}

To investigate the robustness of our study, several sensitivity analyses were conducted. Clinical and cost parameters were varied from $10-20 \%$ more or less from their original value to investigate the impact they would have on the results and to confirm which parameter has the highest impact on our conclusion.

\section{Results}

The estimated target population of 120,574 T2DM adult patients associated with CV risk in Egypt were modelled in the budget impact analysis to compare treatment with liraglutide and sitagliptin, both in addition to the standard of care. The annual results from the perspective of the private health insurers over three-year horizon (Figs. 2 and 3) suggests liraglutide use results in EGP232.5 million (\$54 million) budget savings in medical costs while sitagliptin use results in budget increase EGP29 million (\$6.7 million) in medical costs due to increased number of complications associated with sitagliptin. The liraglutide scenario resulted in a significant reduction in nephropathy ( $-1.5 \%$ difference), mortality $(-1.4 \%$ difference), lower event rates for $\mathrm{HF}$, coronary revascularization, $\mathrm{Ml}$, unstable angina pectoris, and stroke $(-0.6 \%,-0.7 \%,-0.8 \%,-0.10 \%$ and $-0.4 \%$ respectively), and low increase in cases of retinopathy $(0.3 \%$ difference). While sitagliptin scenario resulted in increase in CV mortality, severe hypoglycaemia, HF hospitalization and acute pancreatitis $(0.3 \%, 0.3 \%, 0.1 \%$, and $0.1 \%$ respectively), lower event rates for unstable angina pectoris, stroke, $\mathrm{Ml}$ and pancreatic cancer $(-0.2 \%,-0.2 \%,-0.1 \%$ and $-0.1 \%$ respectively).

The budget impact per patient per month (PPPM) for liraglutide is EGP29 (\$6.7), EGP39 (\$9), and EGP49 (\$11.3) in the first, second, and third year, respectively (Table 2). The budget impact PPPM for sitagliptin 
is EGP11 (\$2.5), EGP14 (\$3.2), and EGP18 (\$4.1) in the first, second, and third year, respectively (as shown in Table 3). Furthermore, adoption of liraglutide resulted in 203 fewer deaths and 550 avoided hospitalizations, while sitagliptin resulted in 43 increased deaths and 14 avoided hospitalizations. The treatment costs of liraglutide use are mostly offset by substantial savings due to fewer CV-related events, avoided mortality and avoided hospitalizations over 3-years.

Table 2

Base case results of liraglutide versus without liraglutide (in Egyptian Pounds)

\begin{tabular}{|lllll|}
\hline Parameters & Year 1 & Year 2 & Year 3 & Cumulative \\
\hline Cost of liraglutide Acquisition & $114,072,451$ & $152,096,602$ & $190,120,752$ & $456,289,805$ \\
\hline $\begin{array}{l}\text { Cost of standard of care by } \\
\text { Substitution* }\end{array}$ & $(13,000,266)$ & $(17,333,688)$ & $(21,667,110)$ & $(52,001,064)$ \\
\hline Drug Cost & $101,072,185$ & $134,762,914$ & $168,453,642$ & $404,288,741$ \\
\hline Non-fatal Myocardial Infarction & $(2,220,074)$ & $(2,960,099)$ & $(3,700,124)$ & $(8,880,297)$ \\
\hline Non-fatal Stroke & $(953,906)$ & $(1,271,875)$ & $(1,589,844)$ & $(3,815,625)$ \\
\hline Hospitalization for heart failure & $(3,499,637)$ & $(4,666,183)$ & $(5,832,729)$ & $(13,998,549)$ \\
\hline Coronary Revascularization & $(1,719,736)$ & $(2,292,982)$ & $(2,866,227)$ & $(6,878,946)$ \\
\hline Retinopathy & 217,033 & 289,377 & 361,721 & $\mathbf{8 6 8 , 1 3 1}$ \\
\hline Nephropathy & $(11,703,224)$ & $(15,604,298)$ & $(19,505,373)$ & $(46,812,895)$ \\
\hline $\begin{array}{l}\text { Hospitalization_Unstable Angina } \\
\text { Pectoris }\end{array}$ & $(277,509)$ & $(370,012)$ & $(462,515)$ & $(1,110,037)$ \\
\hline Mortality & $(37,980,744)$ & $(50,640,992)$ & $(63,301,240)$ & $(151,922,975)$ \\
\hline Medical costs & $(58,137,798)$ & $(77,517,064)$ & $(96,896,330)$ & $(232,551,192)$ \\
\hline Total Costs PPPM & 29 & 49 & 39 \\
\hline $\begin{array}{l}\text { PPPM; per patient per month, *we substituted the market share of the glucose lowering drugs with } \\
\text { liraglutide }\end{array}$ & & & & \\
\hline
\end{tabular}


Table 3

Base case results of sitagliptin versus without sitagliptin (in Egyptian Pounds)

\begin{tabular}{|lllll|}
\hline Parameter & Year 1 & Year 2 & Year 3 & Cumulative \\
\hline Cost of sitagliptin Acquisition & $14,324,166$ & $19,098,888$ & $23,873,610$ & $57,296,665$ \\
\hline Cost of standard of care by Substitution* & $(5,823,714)$ & $(7,764,952)$ & $(9,706,190)$ & $(23,294,856)$ \\
\hline Drug Cost & $8,500,452$ & $11,333,936$ & $14,167,420$ & $34,001,809$ \\
\hline MI & $(277,509)$ & $(370,012)$ & $(462,515)$ & $(1,110,037)$ \\
\hline Ischemic Stroke & $(476,953)$ & $(635,938)$ & $(794,922)$ & $(1,907,813)$ \\
\hline Hospitalization for unstable angina & $(555,019)$ & $(740,025)$ & $(925,031)$ & $(2,220,074)$ \\
\hline Severe hypoglycemia & 87,453 & 116,605 & 145,756 & 349,814 \\
\hline CV Death & $8,138,731$ & $10,851,641$ & $13,564,551$ & $32,554,923$ \\
\hline $\begin{array}{l}\text { Hospitalization for heart failure or CV } \\
\text { death }\end{array}$ & 583,273 & 777,697 & 972,121 & $2,333,091$ \\
\hline Acute pancreatitis & 202,139 & 269,519 & 336,898 & 808,556 \\
\hline Pancreatic cancer & $(333,276)$ & $(444,367)$ & $(555,459)$ & $(1,333,102)$ \\
\hline Medical costs & $7,368,839$ & $9,825,119$ & $12,281,399$ & $29,475,358$ \\
\hline Total Costs PPPM & 11 & 14 & 18 & 14 \\
\hline $\begin{array}{l}\text { PPPM; per patient per month, *we substituted the market share of the glucose lowering drugs with } \\
\text { sitagliptin }\end{array}$ & & & & \\
\hline
\end{tabular}

Liraglutide results in a total initial savings of EGP58 million (\$13 million), EGP77 million (\$17 million), EGP96 million (\$22 million) in the first, second and third year in medical costs, respectively due to avoided complications and hospitalizations. The total cumulative savings over the three years from a private health insurance perspective is estimated at EGP232.5 million (\$54 million) (Table 2). While sitagliptin results in a total increased medical cost of EGP7 million (\$1.6 million), EGP9 million (\$2 million), EGP12 million (\$2.7 million) in the first, second and third year, respectively due to increased complications and hospitalizations. The total cumulative medical costs over the three years from a private health insurance perspective is estimated at EGP29 million (\$6.7 million) (Table 3).

\subsection{Sensitivity Analyses}

The results of a one-way deterministic sensitivity analyses (Fig. 4) suggests that the drug acquisition costs of liraglutide and its market share had the largest impact on the liraglutide model results. T2DM prevalence and the target patients diagnosed and treated with T2DM had the largest impact on the sitagliptin model results (Fig. 5). 
We conducted another scenario analysis (without mortality cost) in liraglutide budget impact model, we found that it has the same conclusion but it resulted in budget savings EGP80 million (\$18 million) only instead of EGP232 million ( $\$ 54$ million) if we included the mortality cost.

\section{Discussion}

Liraglutide was approved by the European Medicines Agency in 2009 and is sold in more than 80 countries to treat T2DM patients [10]. Our results demonstrate that the upfront costs of liraglutide $1.8 \mathrm{mg}$ are mostly offset by the budget savings due to fewer CV-related events and premature mortality avoided (550 avoided hospitalizations and 203 avoided deaths, respectively).

Prior analyses on liraglutide use among T2DM patients also reported cost-savings. In a budget impact study conducted from the Algerian healthcare payer's perspective, liraglutide $1.2 \mathrm{mg}$ resulted in costsavings when compared to insulin glargine among patients insufficiently controlled on oral antidiabetics [11]. Authors reported that more patients reached the target $\mathrm{HbA1c}$ level without the need for intensified treatment regimens, unlike basal insulin that in $79 \%$ of cases requires intensification to a basal-bolus regime or twice daily premix therapy, both of which have higher direct costs [11]. A study in Italy used real world market consumption data and found that adding liraglutide versus standard of care increased the cost per patient between $€ 8.04$ - $€ 25.00$. This study did not consider the cost of complications, and thus did not offset the elevated drug acquisition cost with the cost savings from the reduced rates of complications associated with liraglutide [12]. Another study assessed the budget impact as well as the cost-effectiveness of liraglutide versus the standard of care from the US healthcare payer perspective. Over the lifetime of T2DM patients included in the analysis and with confirmed cardiovascular disease or high cardiovascular risk, liraglutide use was budget neutral and cost-effective [13].

In the United Kingdom, a study on the cost-effectiveness of liraglutide (1.2 and $1.8 \mathrm{mg}$, daily) versus dapagliflozin (10 mg, daily) among T2DM patients, concluded that both doses of liraglutide may be costeffective treatment as a second or third addition to standard of care for patients who are not eligible for SGLT-2i therapy [14]. In Italy, a cost-effectiveness study of liraglutide $1.8 \mathrm{mg}$ versus lixisenatide $20 \mu \mathrm{g}$ (both are GLP-1 receptor agonists) for treating T2DM patients unable to reach acceptable blood glucose levels on metformin, concluded that liraglutide $1.8 \mathrm{mg}$ is likely to be cost-effective versus lixisenatide 20 $\mu \mathrm{g}$ in Italian settings [15]. In France, a study comparing liraglutide, sitagliptin and glimepiride as add-ons for patients not reaching the target $\mathrm{HbA} 1 \mathrm{c}$ level found that while all fell below the willingness to pay threshold, liraglutide was the most cost-effective [16]. The findings of this study were of great significance, as they included CV death and all-cause death outcomes, which is the case with our model. Lastly, in Spain a study comparing $1.8 \mathrm{mg}$ liraglutide and sitagliptin as intensifications for patients on metformin above the target $\mathrm{HbA} 1 \mathrm{c}$ levels, concluded that $1.8 \mathrm{mg}$ liraglutide is cost-effective compared to sitagliptin in Spanish settings [17].

When considering ISPOR Special Task Force in defining the elements of value in health care that were not captured in our model due to lack of local data [18], we can find that the addition of liraglutide not only 
provided high quality adjusted life years (QALYs), life years gained (LYSG) and productivity values as innovative treatment of Egyptian T2DM patients when compared to standard of care, but also can provide the following novel health values; value of hope, real option value, adding more value in severity of disease and as a scientific spillover.

Our study was modelled on the best available evidence from the LEADER and TECOS trials. The budget impact model simulated a patient cohort covered by private health insurance in Egypt and integrated local clinical practice and epidemiological inputs validated by an Expert Panel. We also included various sensitivity analyses to ensure the robustness of the model and to detect any uncertainties. Our study was limited by the use of an international clinical trial with results that may not be specific to Egypt. Given that variations exist in treatment patterns between countries [3]. Our results would be strengthened with clinical parameters specific to Egypt. However, such variations may be negligible because the standard of care of Egypt does not differ compared to countries included in the clinical trials, and Egyptian clinical practice are based on international treatment guidelines (American Diabetes Association Guidelines) [19]. Another strength for our study was the inclusion of mortality cost, as our analysis was conducted from private health insurance perspective, and the private insurance companies pay life insurance to the families of the dead patients.

\section{Conclusion}

The adoption of liraglutide resulted in 203 deaths avoided and 550 hospitalizations avoided. Adding liraglutide resulted in a modest budget impact of EGP 29 - EGP 49 PPPM, suggesting that the upfront drug costs were offset by budget savings due to fewer CV-related complications and deaths avoided. While sitagliptin resulted in a budget impact of EGP 11 - EGP 18 PPPM but associated with 43 deaths increased and 14 hospitalizations avoided compared to the standard of care in Egypt from the private health insurance perspective.

\section{Declarations}

\section{Competing interests}

Elsisi G, Abgad A, Zakaria E, Nasif N, Naiem H, Raafat N, Carapinha J. have no conflicts of interest directly relevant to the content of this article. Afify $A$ is employed in Novonordisk Egypt.

\section{Authors' contributions}

Abgad A, Zakaria E, Nasif N, Naiem H, and Raafat N analyzed and interpreted the collected data. Elsisi $\mathrm{G}$ had a major contribution in writing the manuscript and building the model. Carapinha J. helped in writing the manuscript. Afify A collected needed data about treatments cost. All authors read and approved the final manuscript.

\section{Ethics approval and consent to participate}


“Not applicable"

\section{Consent for publication}

“Not applicable”

\section{Availability of data and material}

This article is a budget impact analysis, and thus there are no underlying data used for this research apart from the data extracted from the articles included in this review. Costs data included used to inform the budget impact analysis are available from the corresponding author upon reasonable request.

\section{Funding}

This study was funded by NovoNordisk Egypt

\section{Acknowledgement}

No Acknowledgement

\section{References}

1. Khunti, S., Khunti, K., \& Seidu, S. (2019). Therapeutic inertia in type 2 diabetes: prevalence, causes, consequences and methods to overcome inertia. Therapeutic advances in endocrinology and metabolism, 10, 2042018819844694.

2. Hegazi, R., El-Gamal, M., Abdel-Hady, N., \& Hamdy, O. (2015). Epidemiology of and risk factors for type 2 diabetes in Egypt. Annals of Global Health, 81(6), 814-820.

3. Marso, S. P., Daniels, G. H., Brown-Frandsen, K., Kristensen, P., Mann, J. F., Nauck, M. A., ... \& Steinberg, W. M. (2016). Liraglutide and cardiovascular outcomes in type 2 diabetes. New England Journal of Medicine, 375(4), 311-322.

4. Green JB, Bethel MA, Armstrong PW, Buse JB, Engel SS, Garg J, et al. Effect of sitagliptin on cardiovascular outcomes in type 2 diabetes. N Engl J Med. 2015;373:232-42.

5. Egypt - The World Factbook. (2020). CIA Factbook. https://www.cia.gov/the-worldfactbook/countries/egypt

6. CDC. (2020). National diabetes statistical report. https://www.cdc.gov/diabetes/pdfs/data/statistics/national-diabetes-statistics-report.pdf

7. AXA research, (2019), (unpublished work).

8. Private sector hospitals (2020) (Alaraby hospital, Cleopatra hospital, Nasser Institute and Elkasr Eleiny hospital).

9. Mauskopf, J. A., Sullivan, S. D., Annemans, L., Caro, J., Mullins, C. D., Nuijten, M., Orlewska, E., Watkins, J., \& Trueman, P. (2007). Principles of good practice for budget impact analysis: report of 
the ISPOR Task Force on good research practices-budget impact analysis. Value in health, 10(5), 336347.

10. EMA. Victoza ${ }^{\circledR}$ EU Summary of Product Characteristics. November 2015. Available at: http://www.ema.europa.eu/docs/en_GB/document_library/EPAR__Product_Information/human/001026/WC500050017.pdf

11. Sellam, Y. (2019). Budget impact savings of liraglutide 1, $2 \mathrm{mg}$ versus insulin in algerian t2d patients insufficiently controlled with oral medications. Value in Health, 22, S146.

12. Capri, S., \& Barbieri, M. (2015). Estimating the budget impact of innovative pharmacological treatments for patients with type 2 diabetes mellitus in Italy: the case of liraglutide (GLP-1). Epidemiology, Biostatistics and Public Health, 12(2).

13. Shah, D., Risebrough, N. A., Perdrizet, J., lyer, N. N., Gamble, C., \& Dang-Tan, T. (2018). Costeffectiveness and budget impact of liraglutide in type 2 diabetes patients with elevated cardiovascular risk: a US-managed care perspective. ClinicoEconomics and outcomes research: CEOR, 10, 791.

14. Vega-Hernandez, G., Wojcik, R., \& Schlueter, M. (2017). Cost-effectiveness of liraglutide versus dapagliflozin for the treatment of patients with type 2 diabetes mellitus in the UK. Diabetes Therapy, 8(3), 513-530.

15. Hunt, B., Kragh, N., McConnachie, C. C., Valentine, W. J., Rossi, M. C., \& Montagnoli, R. (2017). Longterm cost-effectiveness of two GLP-1 receptor agonists for the treatment of type 2 diabetes mellitus in the Italian setting: liraglutide versus lixisenatide. Clinical Therapeutics, 39(7), 1347-1359.

16. Roussel, R., Martinez, L., Vandebrouck, T., Douik, H., Emiel, P., Guery, M., Hunt, B., \& Valentine, W. J. (2016). Evaluation of the long-term cost-effectiveness of liraglutide therapy for patients with type 2 diabetes in France. Journal of Medical Economics, 19(2), 131-144.

17. Pérez, A., Raya, P. M., De Arellano, A. R., Briones, T., Hunt, B., \& Valentine, W. J. (2015). Costeffectiveness analysis of incretin therapy for type 2 diabetes in Spain: $1.8 \mathrm{mg}$ liraglutide versus sitagliptin. Diabetes Therapy, 6(1), 61-74.

18. Lakdawalla, D. N., Doshi, J. A., Garrison Jr, L. P., Phelps, C. E., Basu, A., \& Danzon, P. M. (2018). Defining elements of value in health care-a health economics approach: an ISPOR Special Task Force report. Value in Health, 21(2), 131-139.

19. MOH. (2015). Egyptian Clinical Practice Recommendations for Management of Diabetes Mellitus. Medical Journal of Cairo University.

\section{Figures}




\section{Total adult population $50,364,992$}

\section{T2DM patients $6,889,931$}

T2DM patients diagnosed \& treated (with CV morbidity) $\mathbf{2 , 4 1 1 , 4 7 6}$

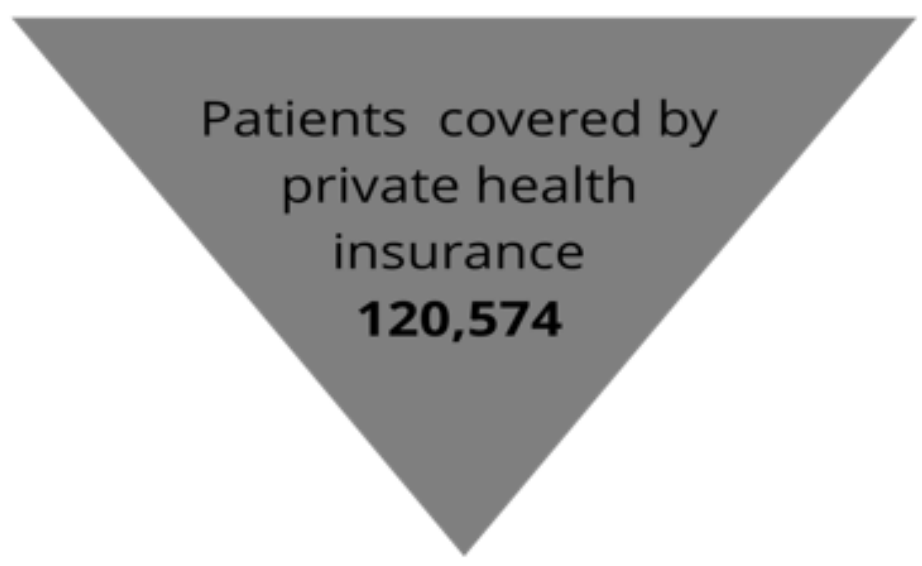

\section{Figure 1}

The Target population T2DM; type 2 diabetes mellitus, CV; cardiovascular 


\section{Liraglutide medical costs}

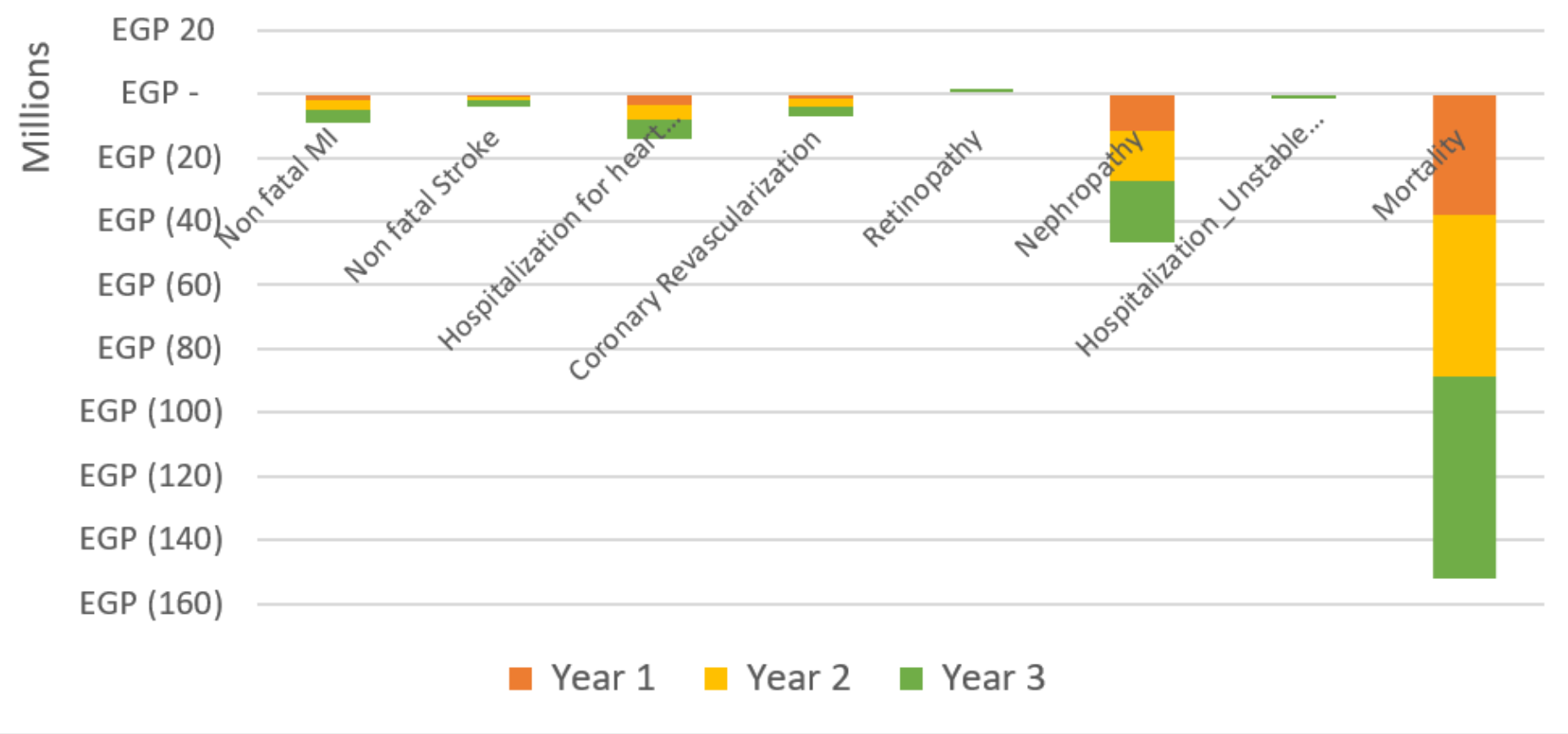

\section{Figure 2}

Liraglutide medical costs over three-year horizon Ml; myocardial infarction

\section{Sitagliptin medical costs}

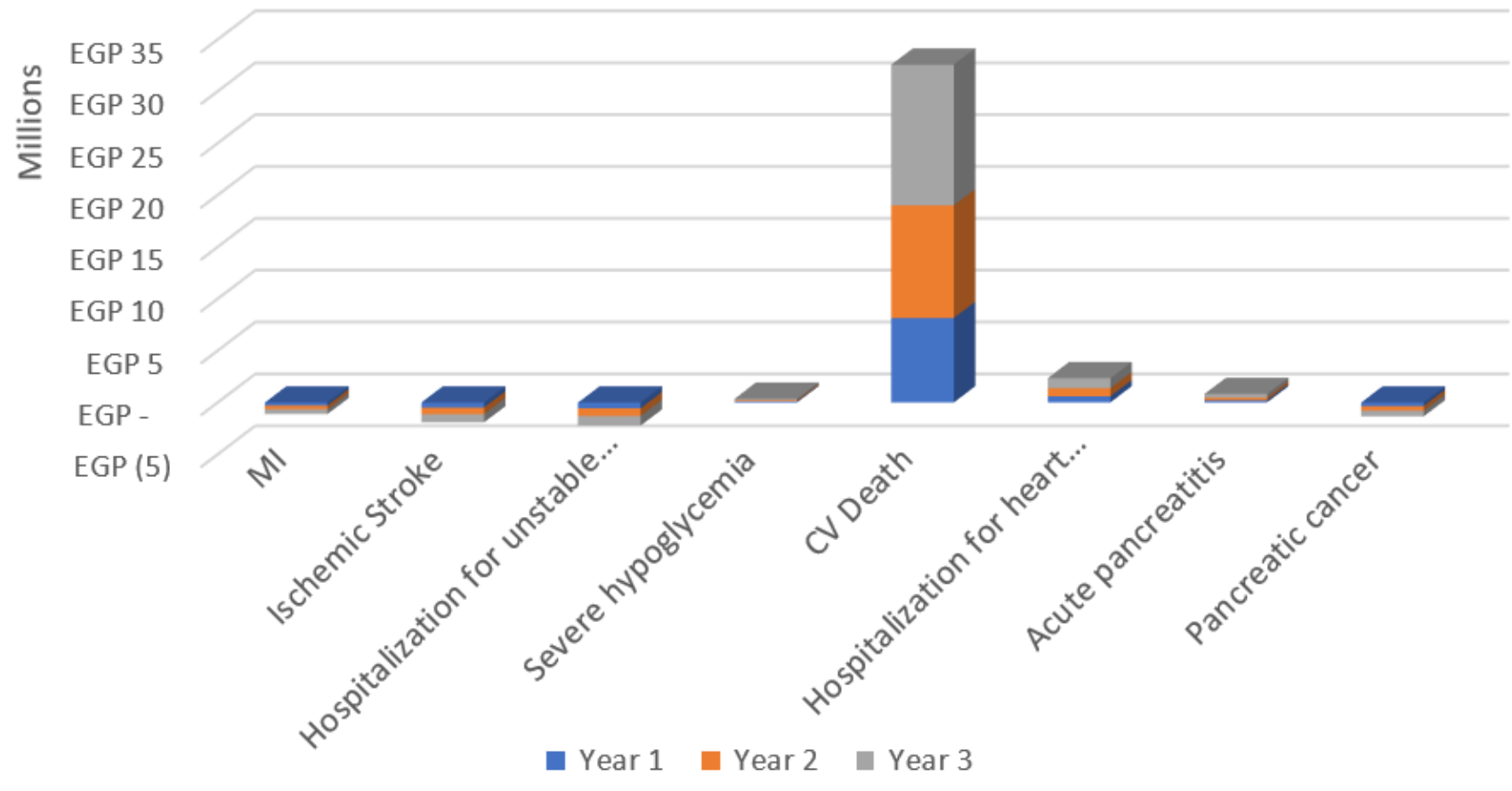

Figure 3 
Sitagliptin medical costs over three-year horizon Ml; myocardial infarction, CV; cardiovascular

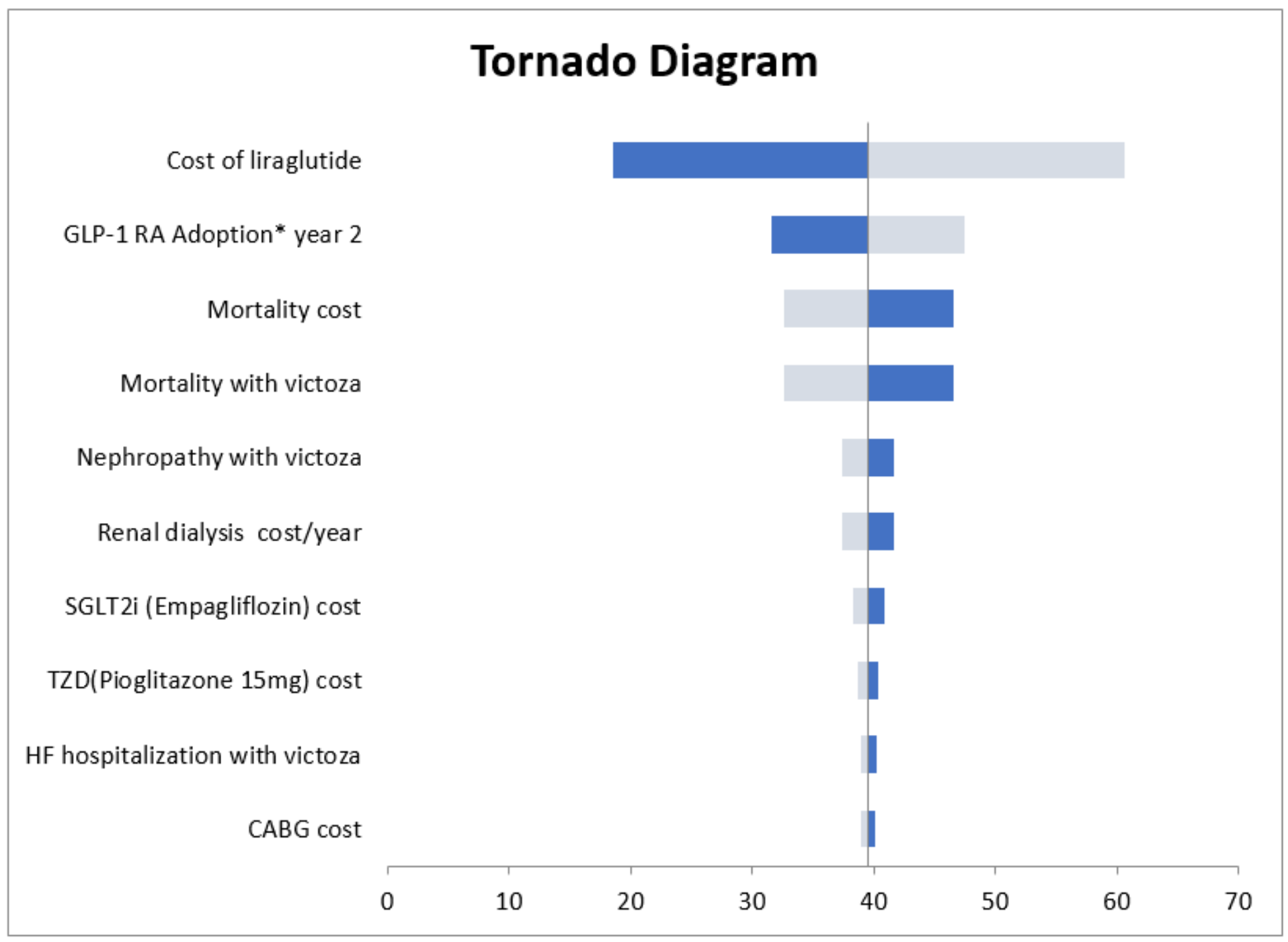

\section{EGP}

\section{Figure 4}

One-way sensitivity analysis results for liraglutide GLP1-RA; Glucagon like peptide 1 receptor agonist, SGLT2i; Sodium/glucose cotransporter-2 inhibitors, TZD; thiazolidinediones, HF; heart failure, CABG; Coronary artery bypass graft The light grey bar corresponds with the upper range, and the dark blue bar with the lower range of an input 


\title{
Tornado Diagram
}

T2DM prevalence

Proportion of people with T2DM diagnosed \& treated

Percentage of patients with CV morbidity

Percentage of patients covered by $\mathrm{PHI}$

Sitagliptin 100 cost

Mortality cost

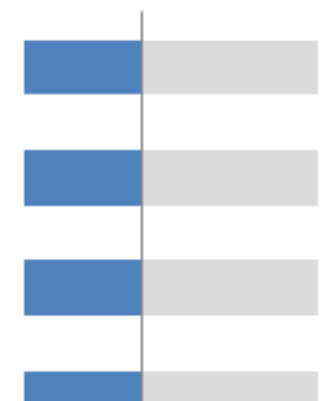

\author{
CV Death_DPP4 \\ DPP4 Adoption* year 2 \\ TZD(Pioglitazone 15mg) cost \\ SU (Gliclazide $60 \mathrm{mg}$ ) cost
}

\begin{tabular}{llllll}
\hline 0 & 5 & 10 & 15 & 20 & 25
\end{tabular}

EGP

\section{Figure 5}

One-way sensitivity analysis results for sitagliptin T2DM; type 2 diabetes mellitus, CV; cardiovascular, PHI; private health insurance, DPP4; dipeptidyl peptidase 4, TZD; thiazolidinediones, SU; sulphonyl urea The light grey bar corresponds with the upper range, and the dark blue bar with the lower range of an input 